\title{
A psicopatologia na formação psiquiátrica
}

Lívia Emy Fukuda1

\section{Resumo}

Em tempo no qual neurobiologia, neuroimagem e o conceito de medicina baseada em evidências dominam o panorama psiquiátrico, busca-se no presente artigo discorrer sobre o papel da psicopatologia na formação de um psiquiatra. Inicia-se situando o momento histórico atual. Em seguida, discorre-se sobre o que se entende por psicopatologia na abordagem fenômeno-estrutural (ou antropológica) e sobre o valor de tal conhecimento na formação do psiquiatra. Finaliza-se o artigo descrevendo uma experiência na escola fenômeno-antropológica chilena, como exemplo que segue a tradição alemã e francesa, nas quais a psicopatologia tem papel fundamental.

Palavras-chave: Psicopatologia; Fenomenologia; Antropologia; Ensino e residência em psiquiatria.

\section{Psychopathology in the psychiatric formation}

\begin{abstract}
Nowadays the knowledge provided by neurobiology, neurosciences and evidence-based studies and experiments dominates the psychiatric panorama. In this article, it is discussed the role of psychopathology in the formation of a psychiatrist in this context. First, it is situated the current historical moment. Then, it is expatiated the psychopathology's concept, mainly, in the structural (or phenomenon-anthropological) approach. The value of such knowledge in the psychiatrist's formation is evaluated afterwards. Finally, it is described an experience in the Chilean phenomenon-anthropological school, as an example that follows the German and French tradition, in which the psychopathology has a fundamental role.
\end{abstract}

Keywords: Psychopathology; Phenomenology; Anthropology; Psychiatric teaching and training residency.

1 Médica psiquiatra formada pela Faculdade de Medicina da USP. Email: liviafukuda@yahoo.com.br 


\section{Panorama atual da psiquiatria: reflexos no ensino}

A psiquiatria é o ramo da medicina que estuda o adoecimento mental, fenômeno heterogêneo e complexo que envolve a relação do sujeito com o mundo. Como especialidade médica, baseia-se em conhecimentos procedentes de vários campos da ciência como biologia, fisiologia, bioquímica, genética, epidemiologia e estatística. Contudo, mantém grande intimidade com as humanidades: filosofia, antropologia, sociologia e psicologia. Abordar tamanha complexidade decorrente das imprecisões e incertezas a respeito da conceituação de seu objeto de estudo, a mente, e da interação desta com o corpo somático e com o mundo torna os limites da psiquiatria mal definidos e alvos de críticas. Pouco se discute sobre a ontologia e epistemologia do domínio mental (Parnas, Sass \& Zahavi, 2012), e isso se reflete diretamente sobre o ensino e o treinamento em psiquiatria.

A transmissão dos conhecimentos psiquiátricos, tendo em vista tais dificuldades e indefinições, acaba por ficar à mercê das ideias predominantes em cada época, dando a impressão de que a psiquiatria tende a progredir em modismos (Ramadam \& Wang, 2011). Ou, conforme, em sentido mais amplo, conclui Messas (2008, p. 215): "Cada período histórico interroga o mundo de modo particular. No seu movimento infinito, a roda da história asperge em cada época histórica um determinado feixe de luz preferencial a iluminar certo aspecto da realidade, à custa do ostracismo de outros".

No momento, a psiquiatria vivencia a temporalidade do agora, imediato e instantâneo. Desejam-se atendimentos velozes e resultados terapêuticos efetivos com menor gasto temporal. A febre atual é buscar ferramentas neurobiológicas e de neuroimagem para fazer diagnósticos precisos de forma imediata. Na mesma medida, a sua espacialidade se dá predominantemente às claras, no aberto e evidente, público. Segue-se quase que exclusivamente e, por vezes, de forma não-crítica, as evidências científicas de forma reducionista, desvalorizando ou até excluindo o íntimo e subjetivo. Há um predomínio da visão de concordâncias, das coletividades, em detrimento das diferenças. O panorama psiquiátrico reflete o que se dá no campo sociológico, no que é prevalente na cultura, na espaço coletivo.

$\mathrm{Na}$ atualidade, observa-se a explosão dos conhecimentos do que se denominam psiquiatria biológica, que inclui neurociências, neuroimagem e psicofarmacologia e há uma maciça valorização do empirismo e do pragmatismo. Tal revolução biológica desloca o foco da psiquiatria para o método nosológico, ou melhor, valoriza-se a doença, não o doente. 
Consequentemente, deparamo-nos com a substituição das detalhadas descrições psicopatológicas das escolas e tradições psiquiátricas doutrinárias pelas entrevistas estruturadas e escalas de sintomas na elaboração dos diagnósticos, com ênfase nos critérios estatísticos representados pelo Manual de Diagnóstico e Estatística (DSM) e pela Classificação Internacional de Doenças (CID). Valoriza-se desproporcionalmente a medicina objetiva, a medicina baseada em evidências, as abordagens baseadas nos sintomas em oposição à subjetividade. E, além da simplificação da linguagem psicopatológica e da forclusão da noção de sujeito (Parada, 2001), verifica-se também a exclusão da hermenêutica do campo psiquiátrico e o ostracismo de conceitos fundamentais como, por exemplo, temporalidade e indeterminação (Messas, 2008).

Não há dúvidas que esse cenário é fruto da necessidade de uniformização e padronização dos diagnósticos (reprodutibilidade e consistência diagnóstica em detrimento da validade diagnóstica), do crescimento da indústria farmacêutica e do reflexo dos avanços da era tecnológica na ciência. Entretanto, deve-se questionar os limites de tamanha objetividade e ter cuidado com reducionismos - sintomatológico e neurobiológico - que antagonizam a complexidade da psiquiatria. Os psiquiatras devem operar em movimento pendular entre o mundo dos fatos (ciência; dimensão biológica) e o mundo dos valores (humanidades; dimensões espirituais, culturais e normativas da coexistência social), a fim de evitar o risco de tornar a disciplina brainless e mindless (Daker, 2012; Volpe \& Sass, 2012). Essa preocupação deve ser questão recorrente na formação dos novos profissionais.

Ainda, seguindo o panorama atual, verifica-se grande alocação dos recursos na psiquiatria biológica e, em muitas partes do mundo, o reconhecimento dos profissionais apenas através de sua produção científica. O ideal seria que a importância e, consequentemente, os investimentos financeiros e humanos fossem igualitariamente divididos entre a assistência aos doentes, o ensino e a pesquisa, mas essa não é a realidade. Assim, nem sempre a formação de novos profissionais médicos é a prioridade de uma instituição. As instituições acabam por determinar se formam médicos ou cientistas, sem se preocupar com as consequências que isso pode vir a ter. Deve-se atentar para o fato de que, como enfaticamente pontua Ramadam (2011, p. 45)

médicos não são cientistas, são profissionais da saúde; Eles não tratam doenças, eles tratam pessoas. $\mathrm{O}$ médico deve responder a uma necessidade expressa do enfermo, e não simplesmente a um estado de doença. Cientistas investigam 
questões clínicas de relevância médica. Médicos se alimentam da ciência para que as informações sejam transformadas em práticas clínicas.

No Brasil - não só na medicina, mas em todo meio universitário - é comum a queixa de que alguns exímios cientistas são professores desinteressados e pouco dedicados, já que para manter a sua função em uma instituição são obrigados a dedicar parte de sua carga horária de trabalho ao âmbito acadêmico. Esse fato influencia diretamente a capacitação dos futuros profissionais, já que a porção teórica pode-se obter nos livros, mas a práxis, a porção arte da medicina e a transmissão de valores só se dão espelhando-se em modelos. Na psiquiatria, em especial, é fundamental o aprendizado e o treinamento do método empático (não somente sua porção intuitiva, préreflexiva, mas principalmente a da empatia de segunda ordem descrita por Stanghellini e Rosfort em 2013).

O viés científico-nosológico se reflete também no mercado editorial e isso, mais uma vez, recai sobre o ensino. O Manual Diagnóstico e Estatístico de Transtornos Mentais da Associação Americana de Psiquiatria encontra-se na quinta edição e é um dos livros mais consultados na área de psiquiatria. Esta última edição foi aguardada com fervorosa ansiedade. Esquece-se que se trata apenas de um manual para padronização de pesquisas epidemiológicas. Em contrapartida, textos clássicos com descrições psicopatológicas minuciosas (relatos de caso, descrições de tipos ideais) tem traduções limitadas ou estão fora de catálogo e não tem divulgação digital. Dessa forma, cria-se uma dificuldade de acesso ao material bibliográfico de qualidade essencial para a formação do bom profissional. Em paralelo, alarmantemente, em muitos cursos de formação em psiquiatria, praticamente se restringe ao estudo de critérios diagnósticos e há uma hipertrofia de disciplinas da moderna psiquiatria biológica na grade curricular em detrimento do aprofundamento em psicopatologia. Ignora-se que a nosologia é apenas um dos métodos para explorar a doença mental. Como consequência, prevalece o caráter técnico do médico, perde-se o sentido da relação médico-paciente, fragmenta-se e torna-se simplista o conhecimento psiquiátrico e desfaz-se a noção de totalidade da existência humana.

Nas palavras de Jaspers (1998, p. 8):

A especialização científica corresponde à remodelação do ensino. Um agregado de disciplinas especializadas entra para o lugar da instrução no pensamento biológico em geral. $\mathrm{O}$ tempo do estudante está tão ocupado pelos planos de estudo que uma conscientização mais profunda é impedida por causa da dispersão na variedade do que há para aprender. Os 
impulsos espirituais da juventude, que carecem de liberdade, são paralisados pela condução do estudo nas andadeiras dos currículos e devido à enorme exigência de memória. Os exames atestam cada vez menos a faculdade de julgar, a qual já nas aulas, de maneira alguma é exercitada em proporção com a quantidade de conhecimentos. Há hoje autênticos e grandiosos modos de conceber a biologia. A tendência geral, porém parece oposta. Em todo o mundo, educam-se pessoas que sabem muito, adquiriram particular habilidade, mas o seu juízo autônomo, o seu vigor para um aprofundamento inquiridor dos doentes é escasso.

O psiquiatra guiado apenas pela perspectiva neopositivista, biológica e cientificista perigosamente tendencia a sua ação à reificação e à despersonalização, pulverizando o paciente e a unicidade de sua existência no anonimato de categorias patogênicas e sistematizações gnosiológicas, todas dentro dos cânones de um pensamento nomológico. Essa postura não dialógica resulta confinada ao horizonte exclusivo e excludente da explicação (no sentido jasperiano), o que é insuficiente para descrever a doença mental no contexto da existência humana (Laing, Phillipson \& Lee, 1966; Manganaro, 2006).

\section{Psicopatologia da formação do psiquiatra}

Inegavelmente devemos agregar os novos conhecimentos à prática e ao ensino em psiquiatria, mas de forma a sempre se manter em diálogo com as fontes psicopatológicas de que se originam e na qual se fecundam. A psicopatologia deve sair da penumbra. Há muitas críticas no sentido de que os estudos psicopatológicos ficaram estagnados no passado, principalmente pelo fato de seu acesso ser difícil ao exigir certo esforço intelectual e pela errônea fama de faltar-lhe uma vocação terapêutica. Necessita-se da retomada da tradição com uma renovação continuada e fértil. O devir da psicopatologia não deve ser a estagnação e muitos estudos já apontam para essa mudança.

Indiscutivelmente, psicopatologia é a disciplina fundamental da psiquiatria. Portanto, na formação do psiquiatra é sine qua non sua presença no currículo acadêmico. E, mais determinante é o significado que se dá ao termo psicopatologia. A definição de psicopatologia apenas em sentido "trivial", como sinônimo de sintoma psiquiátrico, síndrome psiquiátrica, transtorno mental, patologia da psiquê, estudo das doenças mentais é rasa, superficial, sem nuances e ignora a história conceitual do termo. 
Psicopatologia metodologicamente reflete o pensamento sobre conexões estruturais e funcionais da vida interna, especialmente das experiências mentais, normais e anormais. É a disciplina que se ocupa de organizar os diferentes métodos de acesso ao psiquismo.

O desafio do ensino psicopatológico, particularmente do seu ensino prático, se impõe a todos aqueles que consideram que seu intento não se resume a oferecer uma descrição objetiva dos sinais e sintomas, escandida em funções psicológicas, desempenhada por uma espécie de observador ideal, universal, livre de compromissos teóricos e isento de juízos de valor. O modo de operar da psicopatologia traz embutida, como não poderia deixar de ser, uma concepção de saúde e doença e, antes disso, uma ideia acerca do que deve estar subjacente à partilha entre o normal e o patológico (Serpa-Junior, 2007). Questionar o que é doença mental deve ser fundamento para aquele que se aventura no âmbito psiquiátrico. Parece algo óbvio, mas nem sempre há espaço para discutir esse ponto nos centro de formação em psiquiatria.

Stanghellini (2009) distingue, de forma didática, três subáreas da psicopatologia: descritiva, clínica e estrutural. Programas de formação decentes em psiquiatria deveriam contemplar o estudo de todas elas.

A psicopatologia descritiva é definida como a precisa descrição e categorização das experiências anormais como vivenciadas e contadas pelo paciente (perspectiva em primeira pessoa) e observadas em seu comportamento rendimentos). Acessar essas vivências por meio da empatia é considerada a habilidade profissional fundamental do psiquiatra. $\mathrm{O}$ maior nome da psicopatologia descritiva é Karl Jaspers e a escola de Heidelberg. Tem duas principais premissas metodológicas básicas: ausência de teorias apriorísticas e compreensão empática. Como reconhece Jaspers, a fenomenologia é que provê as bases conceituais para exploração das vivências subjetivas, da experiência-vivida. Preconiza-se a descrição do fenômeno assim como ele se apresenta à consciência, como é intuído sem pressuposições teóricas. O método fenomenológico sacrifica a exatidão própria das ciências naturais.

A psicopatologia clínica visa a identificação de sintomas que são significantes na perspectiva da distinção nosográfica e tem como seu maior representante Kurt Schneider (1968). É o elo entre a psicopatologia descritiva e a nosografia. Envolve a tradução de aspectos da expressão e comportamento do paciente em categorias gerais e específicas de sintomas e sinais (perspectiva em terceira pessoa). Interessa-se por eliciar durante a entrevista psicopatológica os fenômenos psíquicos anormais que ajudam o clínico a estabelecer um diagnóstico fidedigno. Os fundamentos do diagnóstico 
criteriológico encontram-se em Schneider. As categorias diagnósticas são importantes no ato clínico principalmente para definição da terapêutica, porém restringir o conhecimento psiquiátrico somente a essa dimensão é uma simplificação irreal e incorre em falhas ao não captar detalhes substanciais da realidade existencial do sujeito.

A psicopatologia estrutural ou fenomenológico-antropológica é representada por Minkowski, Binswanger, Straus, von Gebsattel, Ey, Tellenbach e Blankenburg. Com raízes na psicopatologia descritiva, opera-se também com a noção de fenômeno e não com sintomas. $O$ conceito de sintoma se aplica muito bem à medicina orgânica, somática, pois representa o elo visível de um nexo funcional determinado, cuja presença pressupõe relações causais não visíveis. Quando o nexo funcional é desconhecido, como no caso das doenças mentais, o sintoma passa ter um caráter encobridor e aí vem a necessidade de abrir-se ao fenômeno, ao que se mostra em si e não ao que meramente se anuncia (Dörr, 1995). O fenômeno tem caráter de referência à estrutura. Pela redução fenomenológica, busca-se a captação e a compreensão das estruturas e os modos de organização intencional da consciência (Fuchs, 2002).

Estrutura é, segundo Messas (2006; 2010), a condição de possibilidade da mente consciente, o arcabouço temporo-espacial indispensável para que haja alguma consciência. É algo tão fundamental que apenas pode ser percebido pela sua ausência, alojado nos limites da consciência e não vivenciado diretamente por esta. É primária e anterior aos conteúdos mentais. Forma-se no embate das potencialidades do ser em atrito com a dupla inserção de forças endógenas e inter-humanas históricas. A estrutura serve de fio-guia para dar configuração fenomênica ao pendor profundo do humano ao se voltar para seus semelhantes.

Nessa abordagem, expande-se a psicopatologia descritiva de Jaspers e, consequentemente, a forma como o termo fenomenologia é empregado. Superam-se os axiomas jaspersianos. Aqui, a fenomenologia pode ser pensada como uma aplicação metodológica para descrever as estruturas (categorias) básicas, essenciais do ser-no-mundo, inerentes à experiência consciente, como temporalidade, espacialidade, corporeidade, intencionalidade, intersubjetividade e autoconsciência, assim como para analisar seus possíveis desvios. Busca-se ainda a análise da relação do ser - como existência - com o mundo (situação) e a apreensão das relações de sentido entre a personalidade e a história vital (ou biografia). Visa-se o entendimento do outro como ser autônomo, considerando-se as principalmente as diferenças e não analogias (Stanghellini \& Rosford, 2013). O caráter antropológico se destaca na busca 
pela essência da experiência humana expressa na individualidade, autenticidade, subjetividade, liberdade e historicidade (Kraus, 2003).

Opondo-se às limitações impostas pelas dualidades mente-corpo, sujeito-objeto, saúde-doença e homem-mundo, a orientação fenomenológicoantropológica visa à realização de um diagnóstico holístico, orientado à totalidade do paciente como ser-no-mundo, existência. Significa abordar a doença mental não como uma disfunção da mente, mas como um modo diverso de se manifestar, como uma possibilidade humana (Dörr, 2003). Aqui se evidencia com maior clareza a importante aproximação da psiquiatria à filosofia. Intimidade esta que, ao mesmo tempo em que dá cor e beleza à psiquiatria, é fonte de críticas ferrenhas. E aí surge a questão: aspectos filosóficos e sociológicos devem fazer parte do currículo de formação de um psiquiatra? Para Stanghellini (2004), psiquiatria é filosofia aplicada. Porém, ressalta-se que a aplicação clínico-terapêutica não deve ser perdida de vista. Acredita que noções básicas humanistas são fundamentais no processo formativo em psiquiatria, pois saber como o homem pode ser entendido como existência, como ser social, como espécie, aumenta a cadeia de remetimento (ou referencial) daquele que se defronta com o paciente. A apreensão da totalidade humana de forma integral é impossível (individuo é maior que as categoriais que buscam apreendê-lo), mas se aproxima mais dela à medida que se aumentam as perspectivas de observação do objeto. O psiquiatra pode aumentar as suas possibilidades de aproximação da totalidade real ao combinar os conhecimentos humanistas aos conhecimentos oriundos da psiquiatria biológica.

Um fenômeno constatado em grande parte do mundo é falta de mestres de orientação fenomenológica na posição de catedráticos em instituições psiquiátricas. Isso acaba por dificultar a transmissão destes conhecimentos. Rossi Monti (Rossi Monti \& Cangiotti, 2012) aventa em sua "Crônica de uma oportunidade perdida" quatro pecados capitais da psicopatologia fenomenológica que explicam a sua não inserção nas instituições acadêmicas na Itália. São eles: dogma da não-transmissibilidade (natureza intrínseca da disciplina; exclusivismo e esnobismo dos psicopatólogos), dogma da intraduzibilidade (barreira linguística; incomunicabilidade do conteúdo; bizantinismo linguístico; complicação linguístico-conceitual), marginalização aristocrática (dificuldade de agregação; tendência à diáspora; alergia ao poder e a sua esfera de influência; vocação asceta-apostolar) e dogma da negação da função terapêutica (insidiosa tendência à deriva abstrata, filosófico-contemplativa; recusa à pesquisa empírica com aplicação prática terapêutica). São atualmente raras as instituições que tem uma cátedra de psicopatologia fenomenológica. A seguir, 
é descrita a vivência em uma instituição de orientação fenomenológica, a Escola Fenômeno-Antropológica Chilena.

\section{Escola Fenômeno-Antropológica Chilena}

Dentro da possibilidade de fazer um curso optativo durante a residência médica, tive a oportunidade de conhecer e vivenciar a rotina do Instituto Psiquiátrico "José Horwitz Barak" da Universidad de Chile, em Outubro e Novembro de 2012. Escolhi tal instituição porque havia me interessado pela obra do Prof. Dr. Dörr, "Psiquiatria Antropológica", já que tive o prazer de assistir a sua conferência "Hermeneutic and Dialectic Thinking in Jaspers' General Psychopathology" em setembro de 2011, em Heidelberg (International Conference '100 Years of Karl Jaspers' "General Psychopathology") e o fator língua não seria um empecilho para acessar o psiquismo dos pacientes.

No ano de 2011, nesse serviço foram admitidas 2.791 internações (capacidade de 455 leitos) e realizadas 57.420 consultas ambulatoriais e 25.281 consultas de urgência. Trata-se de um serviço de referência em psiquiatria em todo o Chile e tem orientação fenomenológica.

As instalações do Instituto são bem simplórias. No aspecto material, parecem ter parado no tempo, na época dos grandes manicômios. Enfermarias com quartos grandes que comportam muitos leitos (dez a doze), mobília antiga. Como afirma Parada (2001, p.35): "da predictibilidade de uma ciência está feita a implementação de um ofício". E, a partir disso, esse professor chileno destaca como as influências da ciência e da economia (sistemas de seguridade) podem determinar os tempos de internação e até mesmo como se distribui o espaço arquitetônico de uma organização. Diz:

$$
\begin{aligned}
& \text { se se decide estabelecer tratamento ambulatorial aos } \\
& \text { pacientes e se rechaçam as medidas de internação, muda-se } \\
& \text { completamente o desenho arquitetônico de um centro de } \\
& \text { saúde mental, desde o ponto de vista da estética, da } \\
& \text { funcionalidade, do custo e da distribuição de recursos } \\
& \text { (Parada, 2001, p. 36). }
\end{aligned}
$$

Talvez, a apresentação arquitetônica do local visitado seja reflexo do fato de ser uma instituição de quase 160 anos, que se destaca pelo foco clínico em internações principalmente em fase aguda da doença e, especialmente, pela orientação fenomenológica de seus dirigentes. E, em época em que 
psicopatologia fenomenológica anda fora de moda, recursos para modernização ficam limitados.

O programa de residência médica do Instituto Psiquiátrico "José Horwitz Barak" da Universidad de Chile tem duração de 3 anos e segue o que é preconizado pela World Psychiatric Association (WPA), ou seja, apresenta um currículo didático (ciências básicas, investigação diagnóstica, etiopatogênese, terapêutica, prevenção e promoção de saúde, ética e aspectos legais) e um didático-clínico (rodízios em enfermarias, hospital-dia, ambulatórios, interconsultas, emergência e neurologia), com cargas horárias adequadas. No primeiro ano, o foco são os pacientes de enfermaria e no segundo, os ambulatoriais. No terceiro ano, ocorrem os estágios específicos e os eletivos.

Nessa instituição chilena, o foco é a assistência (como já mencionado) e o ensino. Assim, estive em contato direto com os professores titulares, reconhecidos como mestres da psiquiatria chilena Dr. Otto Dörr Zegers, Dr. Rafael Parada Allende, Dr. Mario Gomberoff Jodorkowsky, e também com Dr. Gustavo Adolfo Murillo Baeza. Observá-los entrevistar e interagir com os pacientes, discutir os casos com aprofundamento psicopatológico, estimular a visão crítica e os questionamentos sobre os limites da foi uma experiência ímpar e enriquecedora. Aqui poder-se-ia impor um certo viés valorativo pela minha inclinação para a abordagem desenvolvida nessa instituição, mas o fato concreto é que durante a visita tive a honra de presenciar uma singela e simbólica homenagem em vida do Instituto a seus três mestres por suas contribuições ao ensino e à assistência. Interessante observar como os becados (forma com denominam os residentes) faziam questão de prestigiar este evento. Hierarquias sempre existirão, mas a possibilidade de trocas eram muito mais abertas.

O professor Dr. Otto Dörr realiza duas atividades semanais. $\mathrm{Na}$ primeira, o docente examina um paciente que não conhece e que é selecionado por sua complexidade ou dificuldades diagnósticas ou terapêuticas. Depois da entrevista, o professor faz uma digressão teórica sobre a enfermidade que aflige o paciente examinado. É um seminário que tem por objetivo ensinar aos residentes como fazer uma entrevista psiquiátrica e um exame clínico rigoroso, deixar surgir o fenômeno, descrever com exatidão a psicopatologia, descobrir as vinculações entre a patologia e biografia, aprender o essencial sobre a patologia do paciente que se apresenta. $O$ segundo seminário semanal é teórico. Neste, fundamentalmente, são revisadas as obras dos grandes representantes da corrente fenomenológicaantropológica, como Karl Jaspers, Hubertus Tellenbach e Wolfgang Blankenburg. Tem por objetivo que os residentes se familiarizem com uma 
corrente de pensamento que exige concentração e vôo teórico e que não se contrapõe a nenhuma outra, senão, ao contrário, as complementa. Estudam-se os axiomas jasperianos e como a orientação antropológico-existencial acabou por superá-los. Adicionalmente, introduz a análise estrutural (apreensão da temporalidade, espacialidade, intersubjetividade, corporeidade), análise situacional (desvelar a reciprocidade pessoa-mundo) e análise existencial (projetos de mundo e condições de possibilidade).

O professor Dr. Rafael Parada é um dos psiquiatras chilenos mais prestigiados que também ministra um seminário de psicopatologia. Parada tem uma visão muito original e nova da disciplina que combina a perspectiva fenomenológica à estruturalista e a da psicanálise lacaniana. Parada (2003, p. 154) afirma: "estruturas psicopatológicas emergem na relação intersubjetiva, fixada na polissemia e variabilidade do discurso transferencial, uma globalidade que resiste às segmentações didáticas e à violência de uma epistemologia que fragmente o encontro temporal com o paciente". Parada é responsável também por estimular o espírito crítico nos alunos e a destacar o valor da psiquiatria social, em especial em relação aos pacientes de longa permanência (crônicos).

O professor Dr. Mario Gomberoff é responsável pela psiquiatria dinâmica e seu seminário visa a ensinar aos residentes o olhar psicodinâmico sem a pretensão de uma formação no preceitos da sociedade psicanalítica. Dr. Gomberoff é dos poucos analistas que permanecem no sistema público de saúde. O conceitos psicanalíticos são ensinados por ele como ferramentas para acessar a subjetividade não só num processo de análise (setting hermético), mas também no contexto de uma consulta clínica hospitalar ou ambulatorial. Há uma abordagem didática teórica (primeiro ano) e uma prática (segundo ano). Com a imposição do domínio de uma psiquiatria quase exclusivamente biológica, acredita-se que é de maior importância que os residentes retomem o aprendizado de se perguntarem também pelo sentido dos sintomas e não somente por sua causa neurobiológica. Estimulase o diálogo da psicanálise com suas fronteiras e com a suas disciplinas afins. Busca-se a retomada da hermenêutica no campo psiquiátrico, com atenção ao uso responsável da interpretação.

O professor Dr. Gustavo Adolfo Murillo trabalhou toda sua vida no Instituto Psiquiátrico e representa a visão da psiquiatria clássica. Trata-se da psiquiatria sobre a qual, em rigor, construíram-se todas as demais, tanto a biológica, como a fenomenológica e a dinâmica. Semanalmente estuda junto com os residentes as obras de Kraepelin, Bleuler, Schneider e Conrad. 
O professor Dr. Eugenio Olea contribui no ensino com seminários de Psicofarmacologia. Introduz a moderna psiquiatria biológica. Como não poderia deixar de constar no currículo, os residentes aprendem a conhecer a química dos fármacos, suas ações, interações, contra-indicações e respectivas indicações nos distintos quadros psiquiátricos.

Semanalmente, há também uma reunião do serviço em que setores (diferentes enfermarias) ficam responsáveis por levar um caso clínico em esquema de rodízio ou se recebe um convidado para uma conferência. Tratase de uma atividade bastante rica, uma vez que é possível ver mais uma vez uma entrevista e uma discussão psicopatológica e terapêutica com debates interessantes. Uma delas por exemplo culminou com o questionamento sobre a ação do psiquiatra. Destaca-se aqui nas próprias palavras do Prof. Dr. Dörr (1995, p. 20):

Cada experiência deve passar pelo fracasso antes de alcançar a sua verdadeira dimensão. Perguntar partindo de uma atitude o mais aberta possível e realizar a experiência da negatividade são ambos elementos substanciais do fazer psiquiátrico tanto prático como científico. Não é possível exercer a vocação de psiquiatra sem saber como perguntar, como fracassar e como resgatar, dialeticamente, algum conhecimento desse mesmo fracasso.

Quando se deseja ter aulas com bons professores, não significa somente que se quer o expert em determinado assunto. De um mestre, não somente se aprende a disciplina particular que esse transmite, senão que o ensino se encontra inscrito em um marco mais amplo que tem que ver com a transmissão de valores fundamentais em que se baseiam a convivência humana e que provê o fundamento de uma prática profissional tão delicada como psiquiatria na qual o que se trata é conviver com outro ser humano na busca da compreensão da particular maneira que se organiza a subjetividade (Maass, Jordán, Tardito \& Vega, 2006).

Prof. Dr. Dörr havia me dito que a melhor época para visitar Santiago seria outubro e novembro, pois, na teoria, encontrar-nos-íamos na primavera chilena. Na prática, o clima não colaborou. O início da estadia foi marcado por um frio anormal e o final por temperaturas típicas de um verão. Porém, em exercício de reflexão durante uma das aula do professor sobre as Elegias 
do Duíno de Rilke², o que concluí foi que vivenciei uma verdadeira primavera interna, uma anunciação e transformação. Fazer psiquiatria voltou a florescer, ter sentido para mim e, consequentemente, para minha prática.

"En ninún sitio, amada, habrá mundo si no es dentro.

Nuestra vida transcurre con transformaciones. Y lo externo,

siempre más insignificante, se desvanece. Donde hubo una vez una casa

duradera, oblicua irrumpe una figura imaginada, algo que pertenece

por completo a lo pensable, como si estuviera aún del todo en el cerebro.

Vastas reservas de energía se crea el espíritu del tiempo,

pero amorfas, como esa fuerza que él extrae de todo.

El ya no conoce templos. Este derroche del corazón

lo atesoramos de modo más íntimo. Sí, y donde aún algo resiste,

una cosa a la que alguna vez se ha rezado, servido y reverenciado,

está ya se ofrece, tal como es, a lo invisible.

Muchos no lo adviertem sin la ventaja, entonces,

de poder construirlo internamente, como pilastras y estatuas más grande! (Sétima Elegia de Rilke)

\section{Referência Bibliográficas}

Daker, M. V. (2012). Revisitando Nobre de Melo: conceitos em psiquiatria. In A. C. T. Rodrigues, L. G. Streb, M. V. Daker, O.D. Serpa (Org.), Psicopatologia Conceitual. (pp. 31-50). São Paulo, SP: Roca.

Dörr Zegers, O. (1995). Psiquiatría Antropológica: contribuciones a una psiquiatría de orientación fenomenológica-antropológica. Santiago de Chile: Universitária.

\footnotetext{
2 Duas vezes por semana, o Prof. Dr. Dörr dá aulas na Universidad Diego Portales. São dois cursos sobre as obras do poeta Rainer Maria Rilke, Soneto de Orfeu e Elegias do Duíno, traduzidas do alemão para o castelhano pelo próprio professor.
} 
(2003). Aproximación al tema del delirio: una posibilidad humana. Archivos de Psiquiatría, 66 (3), 201-212.

Fuchs, T. (2002). The Challenge of Neuroscience: Psychiatry and Phenomenlogy today. Psychopathology, 35, 319-326.

Jaspers, K (1998). O médico na era da técnica. Lisboa, Portugal: Edições 70. (Trabalho original publicado em 1986).

Kraus, A. (2003). How can the phenomenological-anthropological approach contribute to diagnosis and classification in psychiatry? In B. K. W. M. Fulford, K. Morris, J. Z. Sadler, G. Stanghellini (Org.), Nature and narrative: an introduction to the new philosophy of psychiatry (pp. 199-216.). Oxford, UK: Oxford University Press.

Laing, R. D.; Phillipson, H. \& Lee, A. R. (1966). Interpersonal perception. Tavistock, London.

Maass, J., Jordán, J.F., Tardito, S., Vega, J. (2006). Nombramiento del Profesor Mario Gomberoff Jodorkovsky "Maestro de la Psiquiatría Chilena". Revista chilena de neuro-, 44 (1), 63-70.

Manganaro, P. (2006). A psiquiatria fenomenológico-existencial na Itália. Memorandum, 10, 85-92.

Messas, G. P. (2006). Álcool e Drogas: Uma Visão Fenômeno-estrutural. São Paulo: Casa do Psicólogo.

(2008). Psicopatologia Fenomenológica e Psicofarmacologia: um Desafio para a Contemporaneidade. In G.P. Messas, Psicopatologia Fenomenológica Contemporânea (pp. 215-243). São Paulo: Roca. . (2010). Ensaio Sobre a Estrutura Vivida. São Paulo: Roca.

Parada Allende, R. (2001). Patopsicologia y Psicopatologia en la Clinica Psiquiatrica. Santiago, Chile: Mediterraneo.

(2003). Patoplastía en psiquiatría. In: R. Riquelme Véjar, A. Oksenberg Schorr (Ed.). Transtornos de Personalidad. Hacia una mirada integral (pp. 151-156). Santiago, Chile: Sociedad Chilena de Salud Mental.

Parnas, J., Sass, L. A. \& Zahavi, D. (2012). Rediscovering Psychopathology: The Epistemology and Phenomenology of the Psychiatric Object. Schizophrenia Bulletin.

Ramadam, Z. B. A., \& Wang, Y. P. (2011). Psiquiatria e seus Limites. In E.C. Miguel, V. Gentil, W.F. Gattaz (Org.), Clínica Psiquiátrica (pp. 36-48). Barueri, SP: Manole. 
Rilke, R.M. (2010). Las Elégias del Duíno, los Réquiem y otros poemas. Traducción, notas y comentarios de Otto Dörr Zegers. Madrid, Espanha: Visor Libros.

Rossi Monti, M. \& Cangiotti, F. (2012). Maestri senza cattedra - Psicopatologia fenomenologica e mondo accademico. Torino, Italia: Antigone Edizioni.

Schneider, K. (1968). Psicopatologia Clínica. São Paulo, Brasil: Editora Mestre Jou.

Serpa-Junior, O.D. (2007). A inclusão da subjetividade no ensino da Psicopatologia. Interface - Comunic, Saúde, Educ, 11 (22), 207-222.

Stanghellini, G. (2004). Disembodied Spirits and Deanimated Bodies: The Psychopathology of Common Sense. Oxford University Press . (2009). The meanings of psychopathology. Current opinion in psychiatry, 22 (6), 559-64.

Stanghellini, G. \& Rosfort R. (2013). Empathy as a sense of autonomy. Psychopathology, 46 (5), 337-44.

Volpe, U. \& Sass, H. (2012). Why, What and How should Early Career Psychiatrists Learn about Phenomenological Psychopathology? In A. Fiorillo, I. T. Calliess, H. Sass (Org.), How to Succeed in Psychiatry: A Guide to Training and Practice (pp. 82-97). Oxford, UK: Wiley-Blackwell.

World Psychiatric Association. (2002). Institutional program on the core training curriculum for psychiatry. Available from: http://www.wpanet.org/uploads/Education/Educational_Programs /Core_Curriculum/corecurriculum-psych-ENG.pdf. 